\title{
Single Particle CryoEM of the Anthrax Toxin Initial Engagement Complex
}

\author{
Alexandra J Machen ${ }^{1}$, Narahari Akkaladevi², Tommi A White ${ }^{2,3}$, and Mark T Fisher ${ }^{1}$ \\ ${ }^{1 .}$ Department of Biochemistry and Molecular Biology, University of Kansas Medical Center, Kansas \\ City, KS, USA. \\ 2. Department of Biochemistry, University of Missouri, Columbia, MO, USA. \\ ${ }^{3 .}$ Electron Microscopy Core Facility, University of Missouri, Columbia, MO. USA.
}

The lethality of anthrax, a zoonotic disease and bioterrorism agent, is due to the anthrax toxin. This tripartitate toxin consists of protective antigen (PA), lethal factor (LF), and edema factor (EF). The 440 $\mathrm{kDa}$ heptameric PA prepore binds up to three molecules of $\mathrm{LF}$, a mitogen-activated protein kinase kinase protease, and/or EF, an adenylate cyclase. This entire complex, bound to receptor proteins, is endocytosed. As the endosome acidifies to $\mathrm{pH} 5.0$ (late endosome), the receptor bound PA prepore unfolds and refolds into an extend beta-barrel pore structure that penetrates the endosomal membrane. This pore unfolds and translocates the $90 \mathrm{kDa} \mathrm{LF}$ enzyme across the $\mathrm{pH}$ gradient (endosomal $\mathrm{pH} 5.0$ to cytosolic $\mathrm{pH}$ 7.0) of the endosomal membrane through the narrow pore lumen in a $\mathrm{pH}$ driven hypothesized Brownian ratchet mechanism [1]. The translocated LF then refolds on the cytosolic side of the membrane and disrupts cell signaling resulting in cell lethality. To initiate translocation, the Nterminal region of LF moves away from the main body of LF and binds to the alpha clamp region of the PA pore cap [2]. This initial engagement positions the lysine rich $\mathrm{N}$-terminal portion of LF to interact with the 7-fold phe-clamp located further down the PA pore. The directional translocation of LF depends on protonation of acidic residues, the electrostatic character of the PA pore lumen, and remaining positive charges. Subsequent deprotonation of the translocating peptide after the polypeptide passes the phe-clamp region prevents back transfer. To define the nature of the initial engagement and phe-clamp channel blocking steps that occur at different $\mathrm{pHs}$ we are using single particle cryoEM.

With recent advancements of cryoEM, a significant bottleneck in obtaining atomic resolution structures is sample preparation, especially for aggregation prone and membrane proteins like PA [3]. In order to solve the atomic structure of the initial engagement complex, large quantities of soluble PA pore with single bound $\mathrm{LF}_{\mathrm{N}}$ (the N-terminal domain of $\mathrm{LF}$ ) are needed to avoid imposing sevenfold symmetry while obtaining diverse conformational coverage. To this end, $1 \mathrm{LF}_{\mathrm{N}}$-PA pore complexes were produced using an immobilized bead based system and solubilized using authentic lipid bilayer nanodiscs (Figure 1a). E126C $\mathrm{LF}_{\mathrm{N}}$ and PA prepore were incubated prior to immobilization to reduce the number of complexes with multiple bound $\mathrm{LF}_{\mathrm{N}}$ and ensure particle homogeneity. Homogeneous complexes were immobilized by coupling E126C $\mathrm{LF}_{\mathrm{N}}$ to thiol sepharose beads. Beads were rinsed in acid to transition the PA prepores to pores. Pre-nanodisc micelles were then added and bound to the aggregation prone hydrophobic transmembrane beta barrel of PA. The micelles were collapsed into nanodiscs. Soluble complex were released by reducing E126C $\mathrm{LF}_{\mathrm{N}}$. The assembly of $1 \mathrm{LF}_{\mathrm{N}}-\mathrm{PA}$-nanodisc complexes was first confirmed and optimized using negative stain TEM. 1LF N PA-nanodisc complexes were then vitrified to for single particle cryoEM using a FEI Mark IV Vitrobot. Imaging was performed using a FEI Tecnai F30 G2 Twin Transmission Electron Microscope at 200kV. Single particle analysis (EMAN2/SPARX) of cryoEM micrographs clearly indicated 2D class averages with singly bound $\mathrm{LF}_{\mathrm{N}}$. Figure $1 \mathrm{~b}$ highlights side view class averages of $1 \mathrm{LF}_{N}-\mathrm{PA}-\mathrm{Nanodisc}$ complexes. While $\mathrm{LF}_{\mathrm{N}}$ is not visible in all of the averages, our immobilization protocol ensures each complex has at least $1 \mathrm{LF}_{\mathrm{N}}$. Several of the class averages in Figure $1 b$ do not show a distinct nanodisc or PA beta barrel. To create 
expected 2D class averages, a reference model of $1 \mathrm{LF}_{\mathrm{N}}-\mathrm{PA}-\mathrm{Nanodisc}$ complex (Figure 1c) was created in Chimera by docking published structures of $\mathrm{LF}_{\mathrm{N}}$ (PDB 3KWV), PA, excluding domain 4 (PDB 3J9Z), and nanodisc (PDB 2MSC). When filtered to $20 \AA$, back projection of this reference model also had several side views that did not show a distinct $\mathrm{LF}_{\mathrm{N}}$, nanodisc, or beta barrel density (Figure 1d) indicating our sample has the conformational diversity necessary for near atomic to atomic resolution structures.

Our successful assembly of the singly bound $\mathrm{LF}_{\mathrm{N}}-\mathrm{PA}$ pore inserted into a nanodisc will allow us to obtain atomic structures of the initial engagement of LF with the pore prior to translocation. An immobilized bead based system and nanodisc technology allowed us to achieve stable conformationally diverse particles. Now that we have made it through bottle neck of sample preparation, the next step is to collect on a higher powered scope with a direct electron detector. Comparing initial engagement complexes at $\mathrm{pH} 7.5$ and 5 will help us understand how the endosomal $\mathrm{pH}$ facilitates unfolding and directional translocation of the bound LF through the PA translocon.

References:

[1] JAT Young and JR Collier. Annu, Rev. Biochem 76 (2007), 243-265.

[2] D Das and BA Krantz, Proceedings of the National Academy of Sciences (2016), 201600624.

[3] XC Bai, Xiao-Chen, G McMullan, and SHW Scheres, Trends in biochemical sciences 40.1 (2015), 49-57.

[4] The authors acknowledge funding from the Madison and Lila Self Fellowship and KU bridging funds.
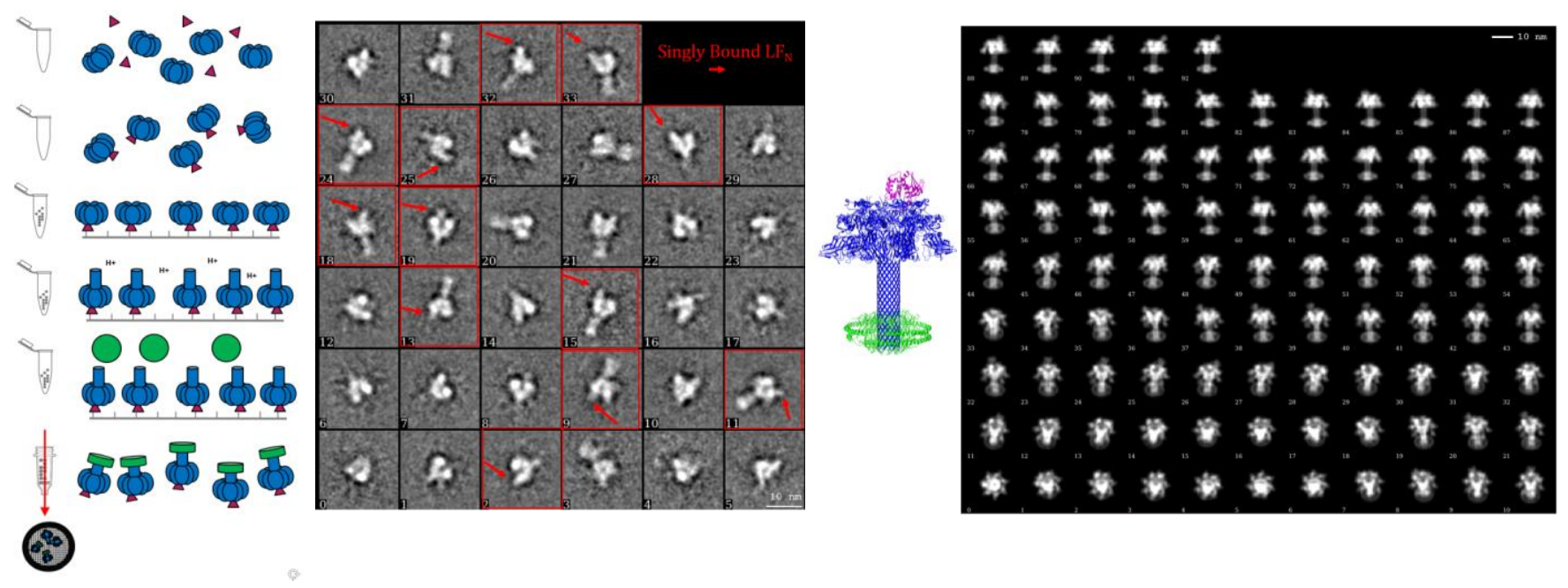

Figure 1. A) Schematic of complex formation. $\mathrm{LF}_{\mathrm{N}}$ (pink triangles) and PA (blue ellipse) complexes were produced using immobilized bead based system and solubilized using lipid bilayer nanodiscs (Green). B) 2D class averages of $\sim 1200$ side view particles using SPARX. Red arrows highlight clear single $\mathrm{LF}_{\mathrm{N}}$ density. Scale bar in bottom right box is $10 \mathrm{~nm}$. C) Side view of reference model of $\mathrm{LF}_{\mathrm{N}}$ (Pink)-PA(Blue)-Nd(Green) complex. D) Projection of $20 \AA$ filtered reference model. Scale bar in top right corner is $10 \mathrm{~nm}$. 